\title{
Tripterygium glycoside protects against puromycin amino nucleoside-induced podocyte injury by upregulating autophagy
}

\author{
JIANGUANG GONG ${ }^{1-3^{*}}$, JUAN JIN ${ }^{1-3^{*}}$, LI ZHAO $^{2,3}$, YIQIAO LI ${ }^{2,3}$, YIWEN LI ${ }^{2,3}$ and QIANG HE ${ }^{1-3}$ \\ ${ }^{1}$ The First Affiliated Hospital of Wenzhou Medical University, Wenzhou, Zhejiang 325035; \\ ${ }^{2}$ Department of Nephrology, Zhejiang Provincial People's Hospital, Hangzhou, Zhejiang 310014 ; \\ ${ }^{3}$ People's Hospital of Hangzhou Medical College, Hangzhou, Zhejiang 310053, P.R. China
}

Received September 14, 2017; Accepted March 22, 2018

DOI: $10.3892 /$ ijmm.2018.3598

\begin{abstract}
Tripterygium glycoside (TG), an active ingredient of the widely used Chinese herb Tripterygium wilfordii Hook $\mathrm{F}$, has immunosuppressive and anti-inflammatory effects. Previous studies have indicated that TG is a potentially effective therapeutic option to treat nephrotic syndrome. The mechanism underlying the therapeutic effect of TG, including its effect on autophagy and apoptosis in podocyte injury, remains to be fully elucidated. The present study aimed to assess the protective effect of TG on podocytes via its potential role in the activation of autophagic and phosphatidylinositol 3-kinase (PI3K) pathways. Using flow cytometry, western blot analysis, cell counting kit- 8 assays and transmission electron microscopy analysis, the effects of TG on puromycin aminonucleoside (PAN)-induced podocyte injury were investigated. Chloroquine (CQ), an inhibitor of autophagy, was used to assess the importance of autophagy in the protective effect of TG. In addition, LY294002, an inhibitor of class III PI3K, was used to identify which signaling pathways TG is involved in. PAN caused marked apoptosis of podocytes, which was significantly antagonized by TG. The expression of microtubule-associated protein 1A/1B-light chain 3 and the appearance of autophagosomes increased significantly following TG treatment, whereas the expression levels of p62 and cleaved caspase-3 were markedly decreased. Podocyte apoptosis decreased significantly when the podocytes were treated with TG compared with the levels of apoptosis in the PAN- and PAN+CQ-treated groups. The expression of phosphorylated AKT was increased significantly in the TG-treated groups, and the effects of TG on the podocytes were significantly inhibited by LY294002. In conclusion, TG protected podocytes from PAN-induced injury,
\end{abstract}

Correspondence to: Dr Qiang He, The First Affiliated Hospital of Wenzhou Medical University, Higher Education Park, Wenzhou, Zhejiang 325035, P.R. China

E-mail: qianghe1973@126.com

${ }^{*}$ Contributed equally

Key words: podocyte, tripterygium glycoside, autophagy, phosphatidylinositol 3-kinase pathway and the effects were attributable to the activation of autophagy, mainly via a PI3K-dependent pathway.

\section{Introduction}

Podocytes are crucial to maintain the normal function of the glomerular filtration barrier. Several kidney diseases, including minimal change disease, focal segmental glomerular sclerosis and membranous nephropathy (1-3), are associated with podocyte injury. Therefore, the treatment of kidney diseases by protecting podocytes has become a popular research topic.

Tripterygium wilfordii Hook F (TwHF), a widely used Chinese herb, is a member of the Celastraceae family of perennial vine-like plants. Tripterygium glycoside (TG), extracted and purified from the root xylem of TwHF, is the active component of TwHF. TG has anti-inflammatory and immunosuppressive effects, and has been used extensively to treat autoimmune and inflammatory diseases, including rheumatoid arthritis, systemic lupus erythematosus and nephrotic syndrome $(4,5)$. For example, TG exhibited promising therapeutic effects on idiopathic membranous nephropathy, which is one of the most common causes of nephrotic syndrome in adults. The injury and apoptosis of podocytes is associated with this typical kidney disease (6-8). In another study of chronic kidney disease (CKD), a systematic meta-analysis showed that therapy with tripterygium preparations significantly decreased proteinuria and serum creatinine levels in patients with CKD (9). However, the mechanism underlying this therapeutic effect remains to be fully elucidated.

In the present study, a puromycin aminonucleoside (PAN)-induced podocyte injury model was used to evaluate the effect of TG on podocyte injury. The study aimed to test the hypothesis that TG protects against PAN-induced podocyte injury by upregulating autophagy via the phosphatidylinositol 3-kinase (PI3K)/AKT pathway.

\section{Materials and methods}

Reagents and antibodies. TG was purchased from Zhejiang Deende Pharmaceutical Co., Ltd. (Zhejiang, China; cat. no. 14002219121). The Annexin V Apoptosis Detection kit was purchased from eBioscience, Inc. (San Diego, CA, USA; cat. no. 88-8007). The Cell Counting Kit-8 (CCK-8) was purchased from Beyotime Institute of Biotechnology (Shanghai,China; cat.no.C0038). Chloroquine(CQ) 
was purchased from Sigma-Aldrich (Merck KGaA, Darmstadt, Germany; cat. no. C6628) and LY294002 was purchased from Selleck Chemicals (Selleck, Houston, TX, USA; cat. no. S1105). The antibodies used in the present study included antibodies against microtubule-associated protein 1A/1B-light chain 3 (LC3)II (cat. no. 12135-1-AP), p62 (cat. no. 18420-1-AP) (both from Wuhan Sanying Biotechnology, Wuhan, China), PI3K [cat. no. Ab151549; Abcam Trading (Shanghai) Co. Ltd., Shanghai, China], AKT (cat. no. Sc-5298; Santa Cruz Biotechnology, Inc., Dallas, TX, USA), phosphorylated (p-)AKT (cat. no. AF0908), caspase-3 (cat. no. 19677-1-AP) and cleaved-caspase-3 (cat. no. 25546-1-AP) (all from Wuhan Sanying Biotechnology).

Cell culture and drug treatment. Conditionally immortalized differentiated mouse podocyte cells (MPC5) were provided by the Cell Resource Center of the Shanghai Institute for Biological Sciences of the Chinese Academy of Sciences (Shanghai, China). The podocytes were cultured in Roswell Park Memorial Institute (RPMI)-1640 medium (cat. no. SH30809.01B; HyClone; GE Healthcare Life Sciences, Logan, UT, USA) supplemented with $10 \%$ fetal bovine serum (FBS; cat. no. 16000-044; Gibco; Thermo Fisher Scientific, Inc., Waltham, MA, USA), $100 \mathrm{U} / \mathrm{ml}$ penicillin G, and $100 \mathrm{mg} / \mathrm{ml}$ streptomycin. The podocytes were maintained and expanded at $33^{\circ} \mathrm{C}$ with $100 \mathrm{U} / \mathrm{ml}$ interferon- $\gamma$ in medium. For podocytes to acquire a differentiated phenotype, the cells were grown under 'restrictive conditions' at $37^{\circ} \mathrm{C}$. To induce injury, podocytes were treated with PAN $(50 \mu \mathrm{g} / \mathrm{ml})$ for $24 \mathrm{~h}$. Different concentrations of TG $(0.31,0.63,1.25,2.5,5$ and $10 \mu \mathrm{g} / \mathrm{ml})$ were added $1 \mathrm{~h}$ prior to PAN treatment. CQ $(20 \mu \mathrm{mol} / \mathrm{l})$ and LY294002 $(20 \mu \mathrm{mol} / \mathrm{l})$ pretreatment were performed $1 \mathrm{~h}$ before PAN treatment.

Cell viability assay. Cell viability was measured using a CCK-8 assay (cat. no. C0038; Beyotime Institute of Biotechnology). The podocytes were seeded in 96-well plates at a concentration of $10^{5}$ cells $/ \mathrm{ml}$. The cells were incubated with different concentrations of TG $(0.31,0.63,1.25,2.5,5$ and $10 \mu \mathrm{g} / \mathrm{ml})$ at $37^{\circ} \mathrm{C}$, $5 \% \mathrm{CO}_{2}$ for $24 \mathrm{~h}$. Following TG treatment, $10 \mu \mathrm{l}$ of CCK-8 was added to each well, and the cells were incubated at $33^{\circ} \mathrm{C}$ for $1 \mathrm{~h}$. The absorbance was detected at $450 \mathrm{~nm}$ using a microplate reader (Thermo Fisher Scientific, Inc., Waltham, MA, USA).

Western blot analysis. The cells were washed three times with ice-cold phosphate-buffered saline (PBS) and lysed with cell lysis buffer (radioimmunoprecipitation assay; cat. no. R0010; Beijing Solarbio Science \& Technology Co., Ltd., Beijing, China), and the total proteins were extracted for western blot analysis. A BCA protein assay (Thermo Fisher Scientific, Inc.) was used for protein quantitation. The soluble material $(50 \mu \mathrm{g})$ was subjected to SDS-PAGE with a $7.0 \%$ acrylamide gel and transferred onto a nitrocellulose membrane by electrophoretic transblotting for 90 min using Trans-Blot SD (Bio-Rad Laboratories, Inc., Hercules, CA, USA). The membranes were blocked at room temperature with 5\% skim milk in Tris-buffered saline Tween-20 for $1 \mathrm{~h}$ and then probed at $4^{\circ} \mathrm{C}$ overnight with the following primary antibodies: Anti-LC3II (1:1,000), anti-p62 (1:500); anti-PI3K (1:600), anti-AKT (1:1,000), anti-p-AKT $(1: 1,000)$ and anti-Caspase-3 (1:500). The membranes were then incubated with the horseradish peroxidase-conjugated goat anti-rabbit secondary antibody (1:1,000; cat. no. A0208; Beyotime Institute of Biotechnology) for $1 \mathrm{~h}$ at room temperature. Following enhanced chemiluminescence detection, the membranes were stripped and proteins were rehybridized with anti- $\beta$-actin antibody $(1: 1,000$, cat. no. 4970) or anti-glyceraldehyde-3-phosphate dehydrogenase (GAPDH) antibody (1:1,000; cat. no. 5174) (both from CST Biological Reagents Co., Ltd., Shanghai, China). The protein levels were determined as the protein/ $\beta$-actin or protein/GAPDH ratio to minimize differences in sample loading. Blots were analyzed using the ImageJ software version 1.8.0-112 (National Institutes of Health, Bethesda, MD, USA).

Analysis of apoptosis by flow cytometry. A fluorescein isothiocyanate-Annexin V Apoptosis Detection kit (BD Biosciences, San Diego, CA, USA) was used to analyze podocyte apoptosis. The cells were washed twice with cold PBS, following which $1 \times 10^{6}$ cells were suspended in $195 \mu \mathrm{l}$ of Annexin V-APC binding buffer. Subsequently, the solution was transferred to a $5 \mathrm{ml}$ culture tube, and $5 \mu \mathrm{l}$ of Annexin $\mathrm{V}$ and $5 \mu \mathrm{l}$ of propidium iodide (PI) were added; the mixture was gently vortexed and incubated for $15 \mathrm{~min}$ at room temperature $\left(25^{\circ} \mathrm{C}\right)$ in the dark. Subsequent flow cytometric analysis (BD Biosciences) was performed within $1 \mathrm{~h}$.

Transmission electronic microscopy. The cell culture media were centrifuged for $10 \mathrm{~min}$ at $714 \mathrm{x} \mathrm{g}$ and $25^{\circ} \mathrm{C}$ The supernatant was discarded and the cells were fixed in $2.5 \%$ glutaraldehyde, dehydrated with graded ethanol, and embedded in Epon 812 using standard laboratory procedures. Ultrathin sections (1 $\mu \mathrm{m})$ were subsequently cut, mounted on nickel grids, and stained with lead citrate for transmission electron microscopy (JSM-IT300LV; JEOL, Ltd., Tokyo, Japan).

Statistical analysis. All statistical analyses were performed using SPSS 19.0 software (IBM SPSS, Armonk, NY, USA). The results are expressed as the mean \pm standard deviation. Group means were compared using one-way analysis of variance followed by the least significant difference test for independent data. All $\mathrm{P}$-values were two-tailed, and $\mathrm{P}<0.05$ was considered to indicate a statistically significant difference.

\section{Results}

Cytotoxic effect of TG on podocytes. CCK-8 and Annexin V/PI assays was performed to detect the cytotoxic effect of TG on the cultured podocytes. The results showed that podocyte viability declined with increasing concentrations of TG. At a concentration of $2.5 \mu \mathrm{g} / \mathrm{ml}$, podocyte viability was significantly decreased (Fig. 1A). Similarly, the percentage of apoptotic cells was increased when treated with TG at $2.5 \mu \mathrm{g} / \mathrm{ml}$ (Fig. 1B). No apparent effects of TG on the proliferation or apoptosis of podocytes were observed below a concentration of $2.5 \mu \mathrm{g} / \mathrm{ml}$.

Protective effect of TG on PAN-induced podocyte apoptosis. To observe the effects of TG on PAN-induced podocyte apoptosis in the present study, podocyte apoptosis was examined using flow cytometry and western blot analysis following treatment with PAN and different concentrations of TG. The in vitro experiments showed that, compared with the control group, the number of apoptotic cells was significantly increased in the PAN group $(\mathrm{P}<0.001)$. In the PAN+TG groups, apoptosis was inhibited by TG when the dose of TG added was $\geq 0.63 \mu \mathrm{g} / \mathrm{ml}$, compared with that in the PAN group (Fig. 2A and B). The 

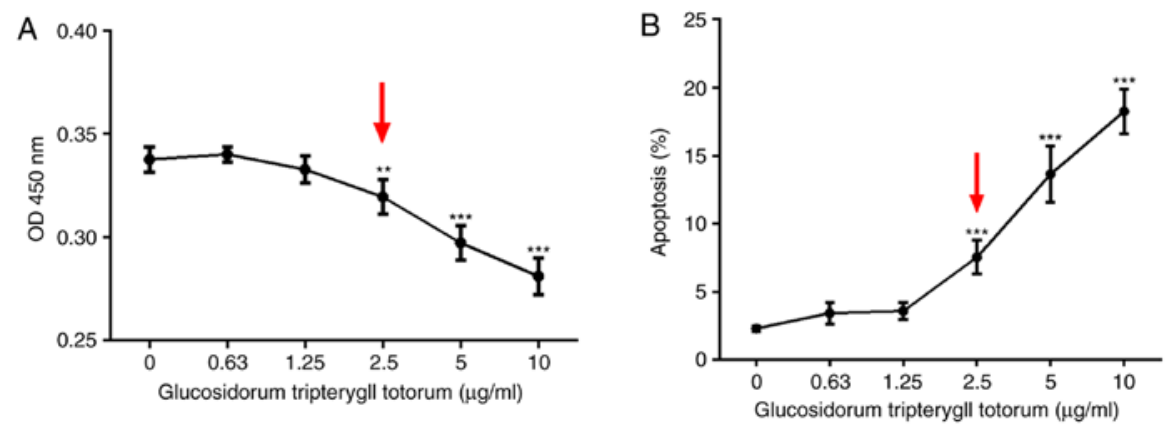

Figure 1. Cytotoxic effect of TG on cultured podocytes. (A) Cell counting kit-8 assay results of MPCs following treatment with different TG concentrations. At a TG concentration of $2.5 \mu \mathrm{g} / \mathrm{ml}$, cell viability was significantly decreased ( $\mathrm{P}<0.01$; red arrow). (B) Apoptotic rates of MPCs following treatment with different TG concentrations, as detected by the Annexin V/propidium iodide assay. The percentage of apoptotic cells increased significantly at a TG concentration of $2.5 \mu \mathrm{g} / \mathrm{ml}(\mathrm{P}<0.001$; red arrow). Data are presented as the mean \pm standard deviation $(\mathrm{n}=3){ }^{* * *} \mathrm{P}<0.01$ and ${ }^{* * * * *} \mathrm{P}<0.001 \mathrm{vs} .0 \mu \mathrm{g} / \mathrm{ml}$ group. MPCs, mouse podocyte cells; TG, tripterygium glycoside.
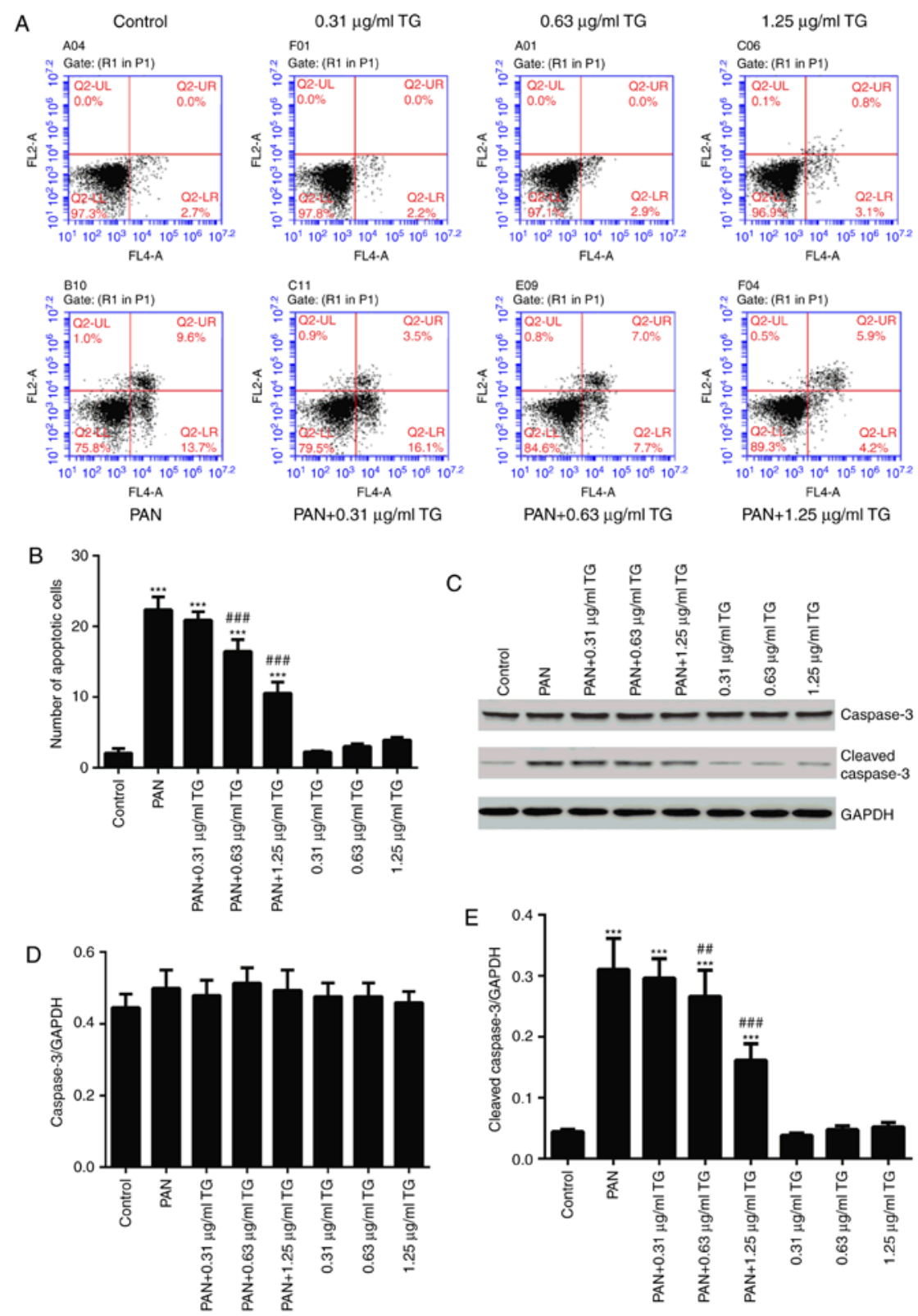

Figure 2. Protective effect of TG on the apoptosis of PAN-treated podocytes. (A) Flow cytometric dot plots of Annexin V and propidium iodide staining. (B) Bar graph showing the percentage of cells undergoing early apoptosis. (C) Representative western blots of cleaved-caspase-3 and caspase-3 in each group. (D) Bar graph showing the relative expression of caspase-3. No significant differences between the groups were observed. (E) Bar graph showing the relative expression of cleaved-caspase-3. The expression of cleaved-caspase-3 was markedly increased in the PAN group, compared with that in the control group, and was decreased significantly in the PAN $+0.63 \mu \mathrm{g} / \mathrm{ml} \mathrm{TG}$ group and PAN $+1.25 \mu \mathrm{g} / \mathrm{ml} \mathrm{TG}$ group, compared with that in the PAN group. Data are presented as the mean \pm standard deviation $(\mathrm{n}=3) .{ }^{* * * *} \mathrm{P}<0.001$ vs. control group; ${ }^{\# \#} \mathrm{P}<0.01$ and ${ }^{\# \# \#} \mathrm{P}<0.001$ vs. $\mathrm{PAN}$ group. TG, tripterygium glycoside; PAN, puromycin aminonucleoside. 
A

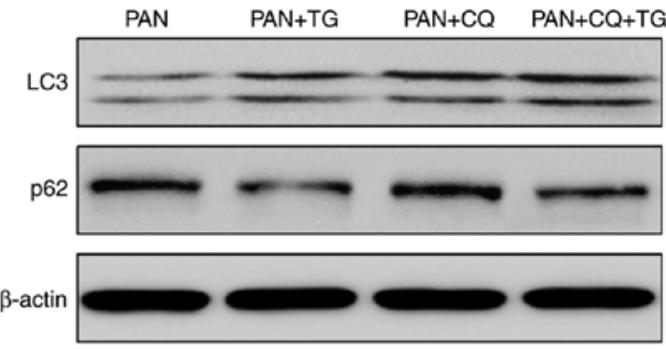

B

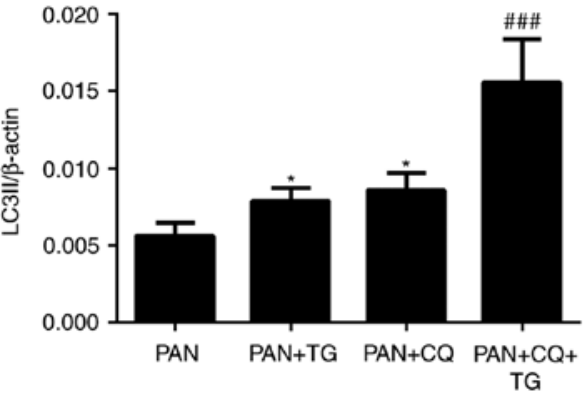

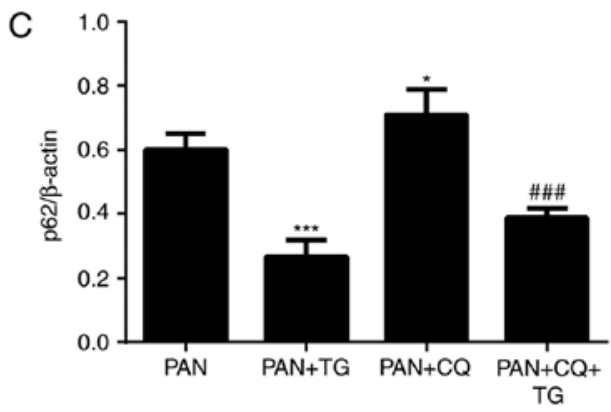

Figure 3. Effects of TG on the autophagy of podocytes. The levels of autophagic markers were detected by western blot analysis. (A) Representative bands of LC3 and p62 in the individual groups. Bar graphs show the relative protein expression of LC3II and p62. (B) TG and CQ promoted the expression of LC3II in podocytes. (C) Expression of p62 was markedly decreased in the PAN+TG group and PAN+CQ+TG group, compared with that in the PAN group and the PAN+CQ group, respectively. Data are presented as the mean \pm standard deviation $(n=3)$. ${ }^{*} \mathrm{P}<0.05$ and ${ }^{* * * *} \mathrm{P}<0.001$ vs. $\mathrm{PAN}$ group; ${ }^{\# \# \#} \mathrm{P}<0.001$ vs. $\mathrm{PAN}+\mathrm{CQ}$ group. TG, tripterygium glycoside; PAN, puromycin aminonucleoside; $\mathrm{CQ}$, chloroquine.

levels of cleaved-caspase-3 and caspase-3 were also detected by western blot analysis to evaluate the apoptosis of the podocytes in all groups. As shown in Fig. 2C-E, the level of cleaved-caspase-3 was increased significantly in thePAN-treated group, compared with that in the control group, whereas TG treatment significantly decreased the level of cleaved-caspase-3 in the PAN-treated podocytes.

TG protects against podocyte injury by upregulating cell autophagy. To evaluate the role of autophagy in the protective effect of TG against PAN-induced podocyte injury, the expression of autophagic markers, LC3II and p62, in podocytes were detected by western blot analysis following treatment with PAN, TG and chloroquine (CQ), a widely used inhibitor of autophagy. As shown by the levels of LC3II and p62, a protein degraded by autophagy pathways, autophagy was activated in the podocytes following treatment by TG and was inhibited by CQ treatment (Fig. 3).

The apoptosis of podocytes was detected by Annexin V/PI following treatment with PAN, TG and CQ. As shown in Fig. 4, the apoptotic rate of the podocytes was negatively correlated with the level of autophagy. The apoptosis of podocytes induced by PAN treatment was reversed by TG treatment. When CQ was added, the protective effect of TG was inhibited. Therefore, an apparent increase in apoptosis in the PAN+CQ+TG group was observed. These results suggested that $\mathrm{TG}$ protected the podocytes from apoptosis by upregulating autophagy.

Assessment of autophagosomes by transmission electron microscopy. Transmission electronic microscopy was used to observe the effect of PAN and TG on autophagy. As shown in Fig. 5, in the control group, the membrane surface of the podocytes had protrusions, the nuclei were irregular, the endoplasmic reticulum structure was clear and autophagosomes were visualized in the cytoplasm. However, in the PAN-treated podocytes, the cytoplasm contained a large number of vacuoles and few autophagosomes. The number of autophagosomes in the cytoplasm of the podocytes increased when treated with TG.

TG induces autophagy by activating the PI3K III signaling pathway. The class III PI3K inhibitor, LY294002, was used to assess the relevance of PI3K pathway activation in the protective effect of TG. The results of the western blot analysis showed that the level of p-AKT was reduced by PAN treatment and was rescued by TG treatment. The level of p-AKT was significantly reduced by LY294002 treatment (Fig. 6A and B) in the LY294002+PAN and LY294002+TG+PAN groups. However, no statistically significant difference was found in the levels of AKT when the podocytes were treated with PAN and TG (Fig. 6A and C). The levels of autophagy makers, LC3II and p62, were also measured by western blot analysis. The level of autophagy was also reduced by PAN treatment and rescued by TG treatment. Treatment with LY294002 also decreased the level of autophagy in the podocytes (Fig. 6A, D and E). The levels of apoptosis of the podocytes were measured by Annexin V/PI analysis. The protective effect of TG on PAN-induced podocyte injury was counteracted by LY294002 treatment (Fig. 6F).

\section{Discussion}

Previous studies have revealed that TG has therapeutic effects against various nephropathies, including IgA nephropathy, diabetic nephropathy and membranous nephropathy. Treatment with the autophagy activator rapamycin has been 
A
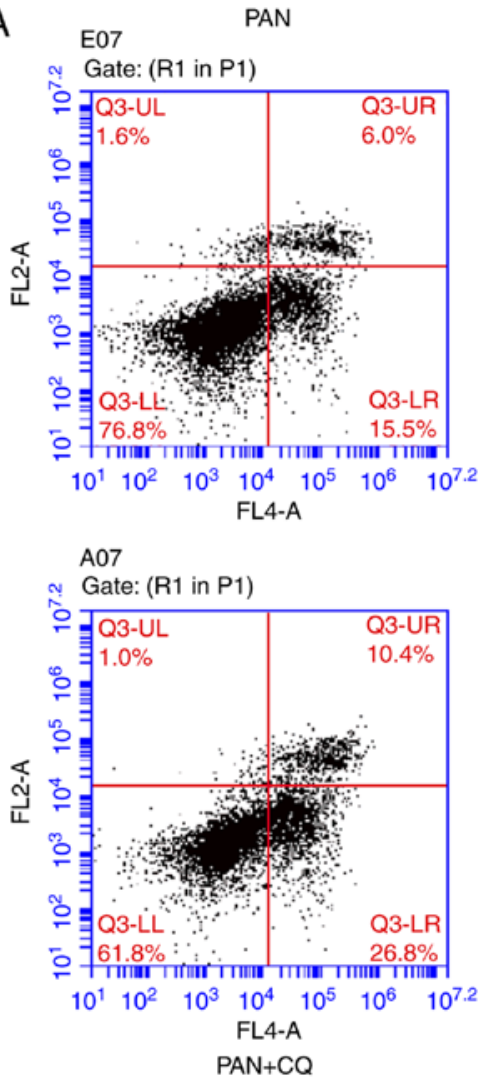

F08 PAN+TG

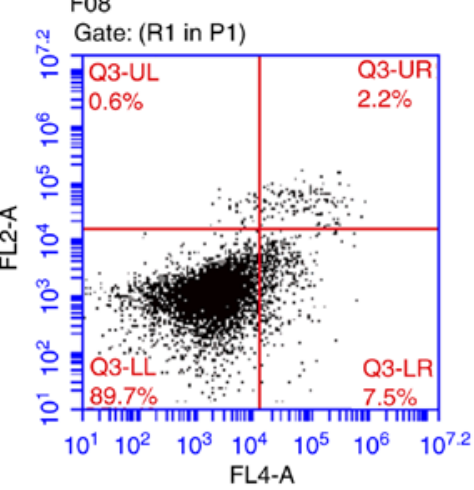

B08

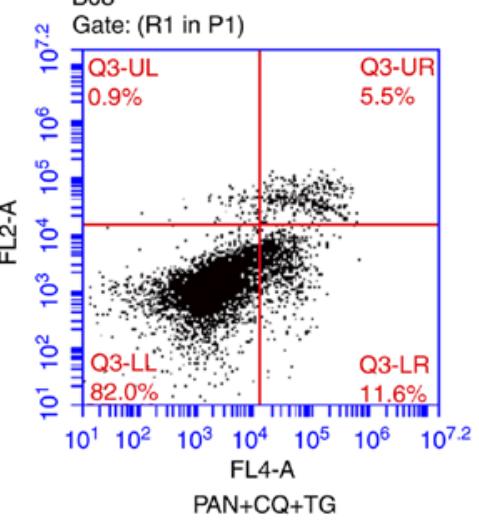

B

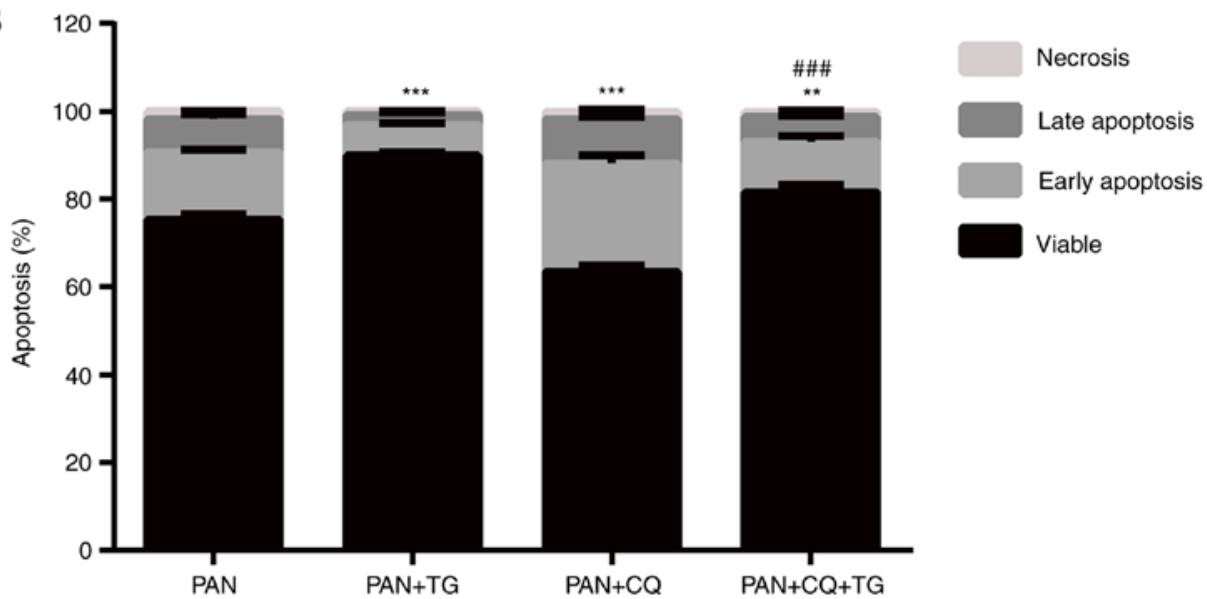

Figure 4. TG protects podocytes from apoptosis through the upregulation of autophagy. (A) Flow cytometric dot plots of Annexin V and propidium iodide staining. (B) Bar graph showing the rates of apoptosis of podocytes in the individual groups. The podocyte apoptotic rate was decreased in the PAN+TG group and the $\mathrm{PAN}+\mathrm{CQ}+\mathrm{TG}$ group, compared with the rate in the PAN group and PAN+CQ group, respectively. Data are presented as the mean \pm standard deviation $(\mathrm{n}=3) .{ }^{*} \mathrm{P}<0.05,{ }^{* * *} \mathrm{P}<0.01$ and ${ }^{* * * *} \mathrm{P}<0.001$ vs. $\mathrm{PAN}$ group; ${ }^{*} \mathrm{P}<0.05,{ }^{\# \#} \mathrm{P}<0.01$ and ${ }^{\# \# /} \mathrm{P}<0.001$ vs. $\mathrm{PAN}+\mathrm{CQ}$ group. TG, tripterygium glycoside; PAN, puromycin aminonucleoside; $\mathrm{CQ}$, chloroquine.

reported to reduce the progression of proteinuria and alleviate pathological lesions in IgA nephropathy rats (10).

Podocytes, as post-mitotic differentiated cells, show limited capacity for regeneration once they are damaged. Podocyte injury can lead to marked proteinuria, and cause several glomerulopathies, beginning with glomerulosclerosis and chronic progression, and eventually leading to end-stage renal disease. The protection of podocytes is one of the most important therapeutic targets for nephrotic syndrome. A number of studies have been performed to examine the therapeutic effect of TG on podocyte injury $(8,11)$; however, the mechanisms remain to be fully elucidated.
In the present study, the classical PAN-induced podocyte injury model was used to evaluate the effect of TG on podocytes. As shown in a previous study (12), PAN induced the apoptosis of podocytes. TG treatment resulted in a reduction in podocyte apoptosis. Further investigation indicated that TG treatment effectively upregulated autophagy and increased the number of autophagosomes. In addition, the levels of PI3K/AKT pathway-related proteins were detected in the various groups, and the results revealed that TG treatment significantly increased the level of p-AKT. This suggested that activation of the PI3K/AKT pathway may be important in the 

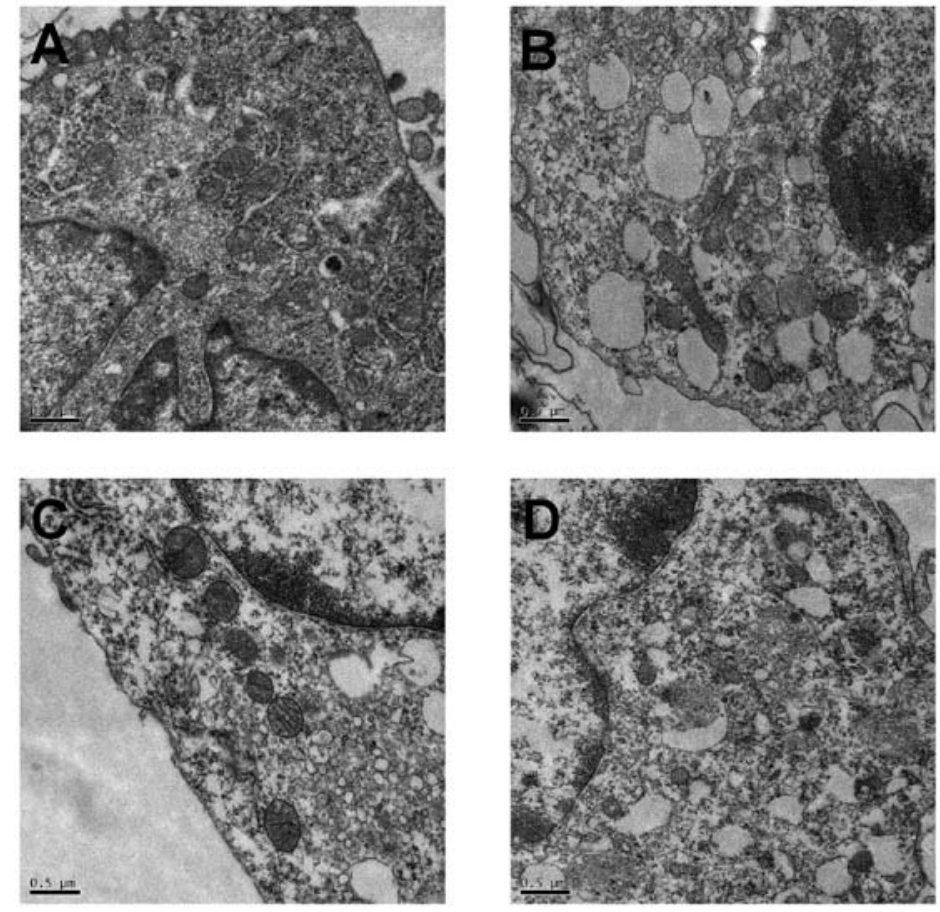

Figure 5. Autophagosomes detected by transmission electron microscopy in the podocytes of individual groups. Autophagosomes were detected by transmission electron microscopy (magnification, $x 20,000$ ). (A) Numerous autophagosomes were observed in the cytoplasm of podocytes in the control group. (B) Compared with the control group, the number of autophagosomes was markedly lower in the PAN-treated podocytes. (C) Compared with the control group, the number of autophagosomes was markedly increased following TG treatment. (D) Compared with the PAN group, the number of autophagosomes was increased following TG treatment. TG, tripterygium glycoside; PAN, puromycin aminonucleoside.
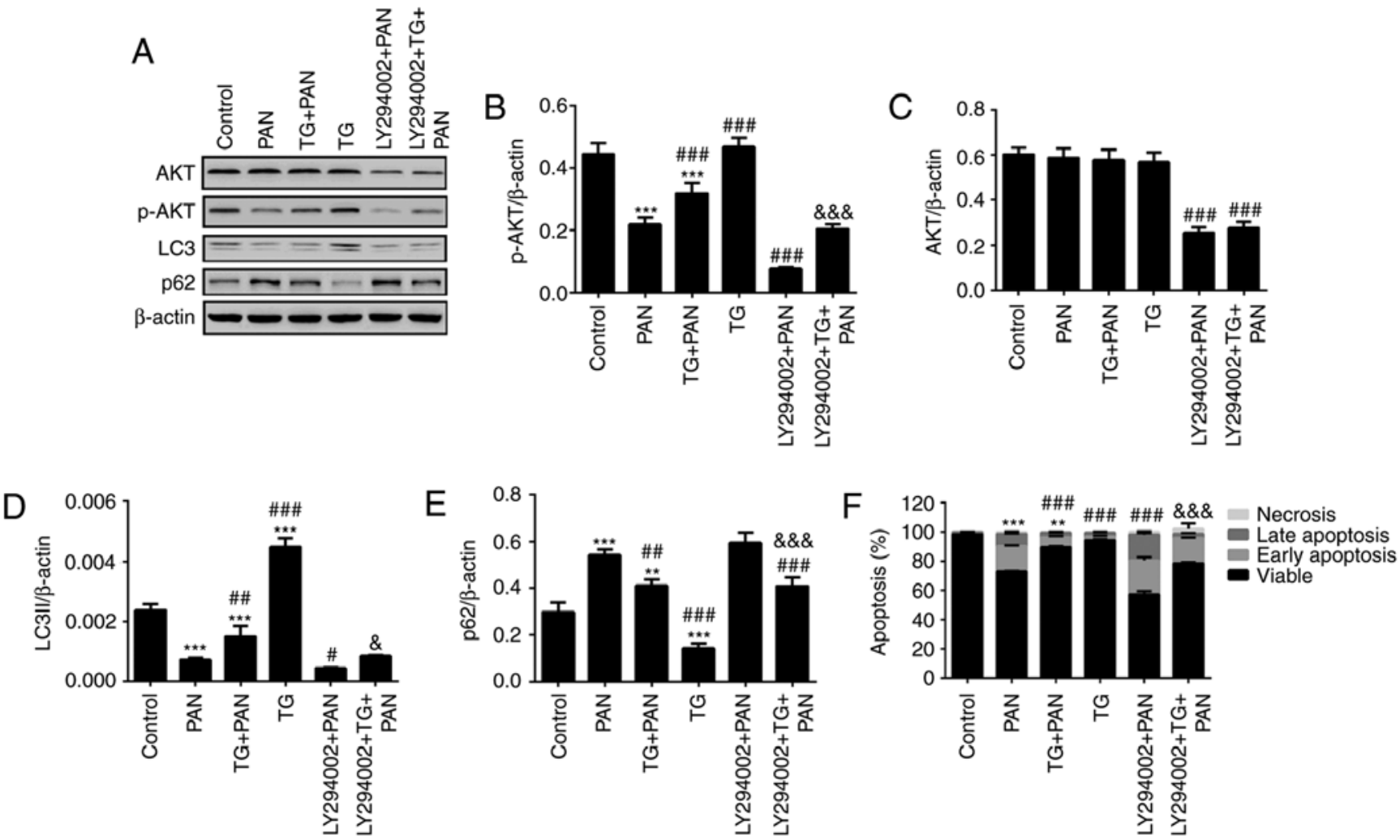

Figure 6. TG promotes the activation of autophagy by PI3K signaling during podocyte injury. (A) Representative bands of AKT, p-AKT, LC3 and p62 proteins in the individual groups. Quantitative evaluation of the levels of (B) p-AKT, (C) AKT, (D) LC3 and (E) p62 in the individual groups. The expression of p-AKT was markedly inhibited by LY294002. The expression of p-AKT was markedly increased in the TG+PAN and LY294002+TG+PAN groups, compared with the expression in the PAN group and LY294002+PAN group, respectively. TG promoted the expression of LC3II and decreased the expression of p62. (F) Apoptotic rates in the groups. Data are presented as the mean \pm standard deviation $(\mathrm{n}=3){ }^{* * *} \mathrm{P}<0.01$ and ${ }^{* * * *} \mathrm{P}<0.001$ vs. control group; ${ }^{*} \mathrm{P}<0.05$, ${ }^{\# *} \mathrm{P}<0.01$ and ${ }^{\# \# \#} \mathrm{P}<0.001$ vs. PAN group; ${ }^{\&} \mathrm{P}<0.05$ and ${ }^{\& \& \&} \mathrm{P}<0.001$ vs. LY294002+PAN group. TG, tripterygium glycoside; PAN, puromycin aminonucleoside; PI3K, phosphatidylinositol 3-kinase; p-, phosphorylated; LC3, microtubule-associated protein 1A/1B-light chain 3. 
regulation of autophagy and in the protective effect of TG in podocyte injury.

Autophagy, a major intracellular lysosomal degradation system, performs homeostatic functions linked to metabolism and organelle turnover. The renoprotective functions of autophagy in podocytes are predominantly mediated by the clearance of affected mitochondria and the removal of protein aggregates, which may trigger inflammation and cell death (13). A previous study suggested that the podocyte foot processes were widely effaced following the prevention of normal autophagic pathways in the nephrons of mice (14). Another study showed that the activation of autophagy ameliorated human podocyte injury induced by high glucose (15). In the present study, it was shown that TG protected podocytes from apoptosis, which was accompanied by increased autophagic activity, as evidenced by an increase in the level of LC3 and a reduction in the level of p62 (Figs. 3 and 4). The effect of TG in upregulating autophagy was confirmed by transmission electronic microscopy. As shown in Fig. 5, the number of autophagosomes in the cytoplasm of podocytes increased significantly when the cells were treated with TG.

The present study also revealed that the level of cellular apoptosis decreased following TG treatment in a dose-dependent manner within the safe concentration range (Figs. 1 and 2). The podocytes were treated with different concentrations of TG and no marked induction of apoptosis was observed in the normal podocytes when the concentration of TG was $<2.5 \mu \mathrm{g} / \mathrm{ml}$, whereas TG markedly inhibited PAN-induced podocyte apoptosis. These results suggested that the optimal dose of TG was able to ameliorate the injury induced by PAN. CQ, a well-known inhibitor of autophagy, was used to verify the role of TG-induced autophagy. Analysis of the protein levels of LC3-II and p62 revealed that CQ inhibited TG-induced autophagy. TG and CQ increased the levels of LC3; however, CQ inhibited the binding process of autophagosomes and their substrate proteins, resulting in the accumulation of p62. Furthermore, apoptosis increased when CQ inhibited autophagy in the PAN group at $24 \mathrm{~h}$. In particular, early apoptosis significantly increased, which may be associated with the duration of cultivation. However, the rates of apoptosis and necrosis were markedly alleviated by TG, and accompanied by the activation of autophagy. It has been reported that autophagy eliminates dysfunctional mitochondria to decrease the generation of cytochrome $c$, which activates apoptosis (16). Autophagy maintains the function of the endoplasmic reticulum via the digestion of protein aggregates and misfolded proteins, thereby inhibiting apoptosis (17). In the future, investigations aim to examine how TG upregulates autophagy and protects against podocyte apoptosis.

PAN increased the generation of reactive oxygen species and activated the cytochrome $c$-caspase-9-caspase-3 apoptotic signaling pathway $(18,19)$. The cysteinyl aspartate specific proteinase (caspase) family of proteases are pivotal in the execution of apoptosis. They function as initiators and executioners in the process of apoptosis. Caspase-3, a typical effector caspase, is responsible for the cleavage of a number of death substrates and leads to cell death (20). The present study showed that TG at a concentration of $1.25 \mu \mathrm{g} / \mathrm{ml}$ markedly alleviated PAN-induced podocyte apoptosis, accompanied by a reduction in cleaved-caspase- 3 . This indicated that inhibiting the activation of caspase- 3 may be involved in the anti-apoptotic effect of TG.

The PI3K/AKT signaling pathway, a well-known cell survival pathway, is critical in the regulation of cell autophagy and apoptosis $(21,22)$. To determine the contribution of the PI3K/AKT signaling pathway in the TG-mediated anti-apoptotic effect in podocytes, the present study examined the phosphorylation and expression of AKT. The results showed that PAN downregulated the level of p-AKT, accompanied by a reduction in LC3 and an increase in p62, whereas TG significantly upregulated the level of $\mathrm{p}-\mathrm{AKT}$ and the activation of autophagy (Fig. 6). LY294002, a classical PI3K inhibitor, significantly downregulated the levels of PI3K, AKT and p-AKT, and inhibited the autophagic activity of the podocytes. Prospectively, TG significantly improved the inhibition of autophagy and decreased the apoptosis of podocytes (Fig. 6). These data demonstrated that the PI3K/AKT pathway is important in the regulation of TG-mediated podocyte autophagy. In the present study, no significant difference in the level of total AKT was found between cells treated with TG and the untreated cells. This suggested that TG promoted the phosphorylation of AKT, rather than promoting the formation of AKT, when it mediates the autophagy of podocytes.

In conclusion, the findings of the present study indicated that TG provided protection against podocyte injury via apoptosis, and that this effect was mediated by the concomitant activation of autophagy. The PI3K/AKT pathway was shown to be important in the regulation of autophagy and apoptosis. These findings provide novel insights into understanding the effects of TG. In addition to its anti-inflammatory effect, TG can reduce proteinuria and serum creatinine levels in CKD; the results of the present study identified novel therapeutic targets for the treatment of podocytopathy and glomerular diseases.

\section{Acknowledgements}

Not applicable.

\section{Funding}

This study was supported by the Natural Science Foundation of Zhejiang Province (grantnos.LY16H050005 andLQ15H050002), and the Project of Scientific Research Foundation of Chinese Medicine (grant nos. 2015ZA011, 2016ZA023 and 2017ZA008).

\section{Availability of data and materials}

All the data supporting findings are contained within the manuscript.

\section{Authors' contributions}

QH designed the study. YL proofread and checked the manuscript. LZ and YL performed the experiments. JJ performed the statistical analyses. JG wrote the manuscript. All authors read and approved the final version of the manuscript. JG and JJ contributed equally. 


\section{Ethics approval and consent to participate}

Not applicable.

\section{Consent for publication}

Not applicable.

\section{Competing interests}

The authors declare that there are no conflicts of interest.

\section{References}

1. Cara-Fuentes G, Clapp WL, Johnson RJ and Garin EH: Pathogenesis of proteinuria in idiopathic minimal change disease: Molecular mechanisms. Pediatr Nephrol 31: 2179-2189, 2016.

2. Mallipattu SK and He JC: The podocyte as a direct target for treatment of glomerular disease? Am J Physiol Renal Physiol 311: F46-F51, 2016 .

3. Nagata M: Podocyte injury and its consequences. Kidney Int 89 : 1221-1230, 2016

4. Xu X, Li QJ, Xia S, Wang MM and Ji W: Tripterygium glycosides for treating late-onset rheumatoid arthritis: A systematic review and meta-analysis. Altern Ther Health Med 22: 32-39, 2016.

5. Chen Y, Gong Z, Chen X, Tang L, Zhao X, Yuan Q and Cai G: Tripterygium wilfordii Hook F (a traditional Chinese medicine) for primary nephrotic syndrome. Cochrane Database Syst Rev 8 : CD008568, 2013.

6. Petermann AT, Krofft R, Blonski M, Hiromura K, Vaughn M Pichler R, Griffin S, Wada T, Pippin J, Durvasula R, et al: Podocytes that detach in experimental membranous nephropathy are viable. Kidney Int 64: 1222-1231, 2003.

7. Liu S, Li X, Li H, Liang Q, Chen J and Chen J: Comparison of tripterygium wilfordii multiglycosides and tacrolimus in the treatment of idiopathic membranous nephropathy: A prospective cohort study. BMC Nephrol 16: 200, 2015.

8. Chen ZH, Qin WS, Zeng CH, Zheng CX, Hong YM, Lu YZ, Li LS and Liu ZH: Triptolide reduces proteinuria in experimental membranous nephropathy and protects against C5b-9-induced podocyte injury in vitro. Kidney Int 77: 974-988, 2010.

9. Zhu B, Wang Y, Jardine M, Jun M, Lv JC, Cass A, Liyanage T, Chen HY, Wang YJ and Perkovic V: Tripterygium preparations for the treatment of CKD: A systematic review and meta-analysis. Am J Kidney Dis 62: 515-530, 2013
10. Liu D, Liu Y, Chen G, He L, Tang C, Wang C, Yang D, Li H, Dong Z and Liu H: Rapamycin enhances repressed autophagy and attenuates aggressive progression in a rat model of IgA nephropathy. Am J Nephrol 45: 293-300, 2017.

11. Hao L, Pan MS, Zheng Y and Wang RF: Effect of Cordyceps sinensis and Tripterygium wilfordii polyglycosidium on podocytes in rats with diabetic nephropathy. Exp Ther Med 7: 1465-1470, 2014

12. Yu SY and Qi R: Role of bad in podocyte apoptosis induced by puromycin aminonucleoside. Transplant Proc 45: 569-573, 2013.

13. Fougeray $S$ and Pallet $\mathrm{N}$ : Mechanisms and biological functions of autophagy in diseased and ageing kidneys. Nat Rev Nephrol 11: 34-45, 2015.

14. Kawakami T, Gomez IG, Ren S, Hudkins K, Roach A, Alpers CE, Shankland SJ, D'Agati VD and Duffield JS: Deficient autophagy results in mitochondrial dysfunction and FSGS. J Am Soc Nephrol 26: 1040-1052, 2015.

15. Xin W, Li Z, Xu Y, Yu Y, Zhou Q, Chen L and Wan Q: Autophagy protects human podocytes from high glucose-induced injury by preventing insulin resistance. Metabolism 65: 1307-1315, 2016.

16. Goldman SJ, Taylor R, Zhang Y and Jin S: Autophagy and the degradation of mitochondria. Mitochondrion 10: 309-315, 2010.

17. Fernández A, Ordóñez R, Reiter RJ, González-Gallego J and Mauriz JL: Melatonin and endoplasmic reticulum stress: Relation to autophagy and apoptosis. J Pineal Res 59: 292-307, 2015.

18. Yu L, Liu Y, Wu Y, Liu Q, Feng J, Gu X, Xiong Y, Fan Q and Ye J: Smad3/Nox4-mediated mitochondrial dysfunction plays a crucial role in puromycin aminonucleoside-induced podocyte damage. Cell Signal 26: 2979-2991, 2014.

19. Ha TS, Park HY, Seong SB and Ahn HY: Puromycin aminonucleoside increases podocyte permeability by modulating ZO-1 in an oxidative stress-dependent manner. Exp Cell Res 340: 139-149, 2015.

20. Portt L, Norman G, Clapp C, Greenwood M and Greenwood MT: Anti-apoptosis and cell survival: A review. Biochim Biophys Acta 1813: 238-259, 2011.

21. Zhao GX, Pan H, Ouyang DY and He XH: The critical molecular interconnections in regulating apoptosis and autophagy. Ann Med 47: 305-315, 2015.

22. Heras-Sandoval D, Pérez-Rojas JM, Hernández-Damián J and Pedraza-Chaverri J: The role of PI3K/AKT/mTOR pathway in the modulation of autophagy and the clearance of protein aggregates in neurodegeneration. Cell Signal 26: 2694-2701, 2014.

This work is licensed under a Creative Commons Attribution-NonCommercial-NoDerivatives 4.0 International (CC BY-NC-ND 4.0) License. 